University of Nebraska - Lincoln

DigitalCommons@University of Nebraska - Lincoln

April 1979

\title{
Electronic properties and superconductivity of Zr-Pd glasses
}

G.R. Gruzalski

University of Nebraska - Lincoln

J.A. Gerber

University of Nebraska - Lincoln

David J. Sellmyer

University of Nebraska-Lincoln, dsellmyer@unl.edu

Follow this and additional works at: https://digitalcommons.unl.edu/physicssellmyer

Part of the Physics Commons

Gruzalski, G.R.; Gerber, J.A.; and Sellmyer, David J., "Electronic properties and superconductivity of Zr-Pd glasses" (1979). David Sellmyer Publications. 171.

https://digitalcommons.unl.edu/physicssellmyer/171

This Article is brought to you for free and open access by the Research Papers in Physics and Astronomy at DigitalCommons@University of Nebraska - Lincoln. It has been accepted for inclusion in David Sellmyer Publications by an authorized administrator of DigitalCommons@University of Nebraska - Lincoln. 


\title{
Electronic properties and superconductivity of $\mathrm{Zr}-\mathrm{Pd}$ glasses
}

\author{
G. R. Gruzalski*, J. A. Gerber, and D. J. Sellmyer \\ Behlen Laboratory of Physics, University of Nebraska, Lincoln, Nebraska 68588
}

(Received 29 September 1978)

\begin{abstract}
Electrical-resistivity measurements between 1.4 and $300 \mathrm{~K}$ and room-temperature magneticsusceptibility measurements were performed on the metallic glasses $\mathrm{Zr}_{x} \mathrm{Pd}_{1-x}$, for $x=0.59$, $0.63,0.67$, and 0.70 . The samples, prepared by a modified hammer-anvil technique, exhibit negative temperature coefficients of the resistivity at temperatures from about 10 to $300 \mathrm{~K}$. These data can be understood in terms of the extended Ziman theory if it is assumed that $\mathrm{Zr}$ and Pd contribute about 2 and 1 conduction electrons, respectively. Assuming that the magnetic susceptibility is a qualitative measure of the electronic density of states at the Fermi level, $N\left(E_{F}\right)$, it is shown that $N\left(E_{F}\right)$ does not seem to decrease with composition as the eutectic composition is approached. Thus, for this system, there is no obvious correlation between the thermodynamic stability of the liquid phase and the electronic density of states. The superconducting transition temperature $\left(T_{c}\right)$ scales linearly with $x$ and the valence-electron-per-atom ratio $(z)$. However, as in the case for other intertransition-metal glasses composed of distant neighbors in the Periodic Table, $T_{c}(\boldsymbol{z})$ falls below the Collver-Hammond $T_{c}(\mathbf{z})$ curve for amorphous metals and alloys of neighboring metals of the $4 d$ and $5 d$ transition series.
\end{abstract}

\section{INTRODUCTION}

There is at present a great deal of interest in , developing fundamental descriptions of electronic, vibrational, and magnetic excitations in metallic glasses. ${ }^{1}$ Here the term glass means an amorphous solid formed by liquid quenching. The field is a fertile one because so many of the observed properties - for example, electrical resistivity, heat capacity, and magnetic ordering - are drastically different from those observed in crystalline metals or alloys. Under these circumstances it is not surprising that several controversial suggestions have been made to explain the observed phenomena, and that even a qualitative understanding has not been easy to achieve.

In addition to understanding electronic, vibrational, and magnetic states in metallic glasses, a significant problem is understanding the basis for thermodynamic and kinetic stability of these materials. Various models have been advanced to account for the observed structures and the role of composition on the ease of formation and the resistance to crystallization of these glasses. These include models based on (i) the packing of hard spheres, ${ }^{2}$ (ii) chemical-bonding effects, ${ }^{3}$ and (iii) the energetics of the conductionelectron system. ${ }^{4}$ The problem is made more difficult by the fact that quite a number of classes of metallic glasses can be identified. One class consists of a "late" transition or noble metal $T$ combined with a metalloid or glass former $G$. These glasses typically have the form $T_{x} G_{1-x}$ with $x=0.8$. A second class has the form $N_{x} T_{1-x}$, where $N$ is an "early" transition metal, and where $x$ can vary considerably; for example, from about 0.4 to 0.7 . Examples of these intertransition metals are $\mathrm{Zr}-\mathrm{Cu}, \mathrm{Nb}-\mathrm{Ni}$, and $\mathrm{Zr}-\mathrm{Rh}$. A third class of glass-forming alloys has the form $R_{x} T_{1-x}$, where $R$ is a rare earth and $x \approx 0.6$ to 0.7 . Examples of these alloys are $\mathrm{Gd}_{0.65} \mathrm{Co}_{0.35}, \mathrm{Er}_{0.64} \mathrm{Fe}_{0.36}$, etc. A fourth type of glassy alloy is $\mathrm{Mg}_{0.70} \mathrm{Zn}_{0.30}$, which contains only $s$ and $p$ conduction electrons. Finally, metallic glasses of the form $A_{x} \mathrm{O}_{1-x}$ recently have been reported, ${ }^{5}$ where $A$ represents an alkali metal and the oxygen concentration $(1-x)$ is about $15 \%$

In part, the difference between the various models may be a semantic one arising from the approach one takes to understand solids in general. For example, the tension between the "chemical-bond" and "bandstructure" approach has been present during the long history of the study of crystalline metallic compounds and alloys, and recent work suggests that it is continuing in the area of metallic glasses. A connection between the various approaches is suggested, for example, by the extended x-ray-absorption finestructure (EXAFS) measurements of Hayes et al. ${ }^{6}$ on amorphous Pd-Ge alloys. These measurements indicate that each $\mathrm{Ge}$ atom is, on the average, surrounded by $8.6 \pm 0.5 \mathrm{Pd}$ atoms and no $\mathrm{Ge}$ atoms. These investigators thus conclude that there is a great deal of chemical order in transition-metal-metalloid systems of this type. This strong degree of chemical 
ordering had been anticipated on the basis of heats of mixing ${ }^{7}$ and structural models of both glasses and crystals. ${ }^{8}$ Recently Nagel and $\mathrm{Tauc}^{9}$ have shown, using pseudopotential theory based on the nearly-freeelectron (NFE) approximation, that electronic-energy considerations also lead to this type of order. However, recently reported systems, which may not be describable in terms of simple pseudopotential theory, are metallic glasses of oxidized $\mathrm{Rb}$ and $\mathrm{Cs}$. For these glasses, Bauhofer and Simon ${ }^{5}$ argue that there is significant charge transfer from the alkali metal to the oxygen ion. Thus the chemical-bonding model is more appropriate for these materials than a NFE model. The authors also conclude that strongly bonded ionic clusters are responsible for the enhanced tendency for glass formation.

One of the interesting questions regarding glasses such as $\mathrm{Zr}-\mathrm{Cu}$ or $\mathrm{Zr}-\mathrm{Pd}$ is: What effective valence $z$ must be chosen for each element to obtain the correct Fermi surface (which must be spherical in a metallic glass)? The experimental results of Norman and Harris ${ }^{10}$ suggest that in crystalline $\mathrm{Zr}-\mathrm{Pd}$ alloys there is a transfer of electrons from $\mathrm{Zr}$ to $\mathrm{Pd}$. In light of this result, the question regarding effective valence might be rephrased as follows: How many electrons contributed by the $\mathrm{Zr}$ atoms fill $d$ states of $\mathrm{Pd}$, i.e., how much smaller than four is $z_{\mathrm{Zr}}$ ?

It is well established that the ease of glass formation of a liquid increases as the temperature difference, $T_{m}-T_{g}$, decreases. Here $T_{m}$ and $T_{g}$ are the liquidus and glass-transition temperature, respectively. Because $T_{g}$ usually varies relatively slowly with composition, it follows that the ease of glass formation will be greatest at compositions near that of a eutectic. Similarly, the ease of glass formation should increase with an overall lowering of the liquidus curve. ${ }^{11}$ To illustrate how such a lowering might occur, the free energies of an element $A$, a crystalline compound $A B$, and the liquid are sketched in Fig. 1. The dashed line shows what the free energy of the liquid might be if some extra thermodynamic stabilization factor were present. Consider the process of cooling. Since the entropy of the liquid is much larger than that of either the compound or crystal, the free energy of the liquid will increase faster than that of the compound or the crystal with decreasing temperature. Thus, the eutectic temperature (the temperature at which one tangent curve touches all three phases) is reduced by the presence of the extra stabilization factor. The extent to which the eutectic temperature is driven down, and the concomitant lowering of the liquidus curve, will depend on the exact form of the extra stabilization factor. A point of interest is that Nagel and Tauc, ${ }^{4}$ using the NFE model, predict a minimum in the density of states at the Fermi level, $N\left(E_{F}\right)$, when the electronic structure is such that $2 k_{F}=q_{p}$, where $k_{F}$ is the Fermi wave vector and $q_{p}$ the wave number of the first peak in the structure factor. Again, such a thermodynamic stabilization should lead to an overall lowering of the liquidus curve (with respect to that curve which would result if this stabilization factor were absent) and thus increase the tendency of the liquid to form a glass. If this stabilization is maximized in the neighborhood of the eutectic composition (as is suggested by Nagel and Tauc's ${ }^{4}$ result regarding metal-metalloid glasses), then one might expect $N\left(E_{F}\right)$ to decrease with composition as the eutectic composition is approached.

Another point of interest is that the extended $\mathrm{Zi}$ man theory for electrical resistivity of metallic glasses ${ }^{12}$ predicts a negative temperature coefficient (NTC) of the resistivity for concentrations for which $2 k_{F} \simeq q_{p}$. Such NTC values of ten are observed for metal-metalloid and intertransition-metal glasses, thus providing some confirmation of the NFE model for these materials.

The observation of superconductivity in an amorphous material was reported first in $1954 .{ }^{13}$ In the years since, a wide variety of amorphous metals have been found to exhibit superconductivity. ${ }^{14} \mathrm{~A}$ particularly interesting and ambitious experiment was carried out by Collver and Hammond, ${ }^{15}$ who measured the superconducting transition temperature $T_{c}$ of highly disordered vapor-quenched metals and alloys of neighboring metals of the $4 d$ and $5 d$ transition

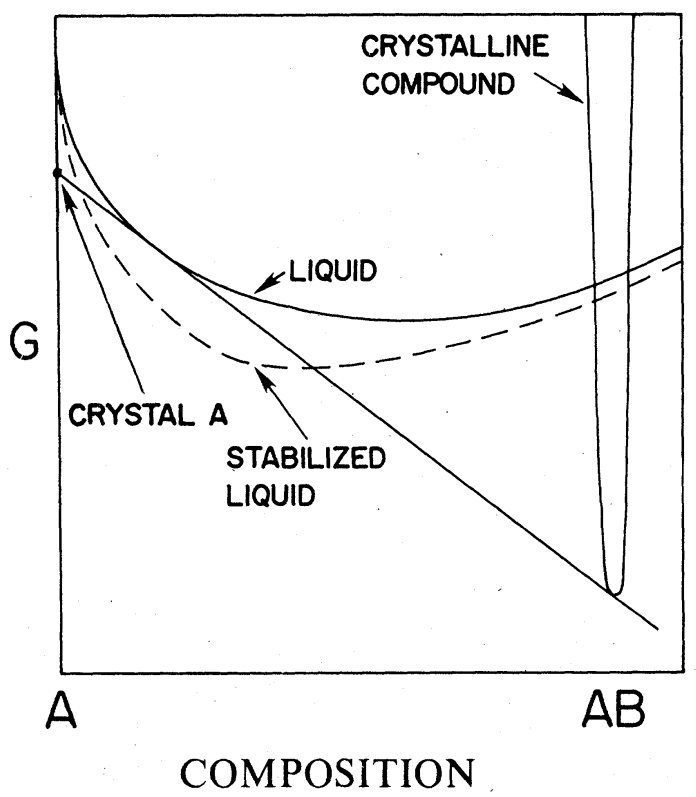

FIG. 1. Gibbs free energy $G$ vs composition for an element $A$, a crystalline compound $A B$, and the liquid. The dashed line shows what the free energy of the liquid might be if some extra thermodynamic stabilization factor were present. The free energies are shown at the eutectic temperature of the system before the extra stabilization factor is operative. 
series. They found that $T_{c}$ varies slowly with the valence-electron-per-atom ratio $(z)$ and has a single broad maximum near the middle of the $d$ series. ( $z$ was determined by considering all the electrons outside of the filled inert-gas core shell as valence electrons.) It has been suggested ${ }^{14}$ that such a variation might correlate well with $N\left(E_{F}\right)$, which varies slowly with $d$-band occupation and also has a broad maximum. An interesting question is how important is the effect of the electronic structure compared with, say, the real-space structure on the superconductivity of metallic glasses.

The purpose of the present work was to examine some of the questions raised above for the $\mathrm{Zr}_{x} \mathrm{Pd}_{1-x}$ system, for $0.59 \leqslant x \leqslant 0.70$. The electrical resistance of several alloys was measured across this composition region to look for NTC and for comparison with the extended Ziman theory. The magnetic susceptibility, which is proportional to $N\left(E_{F}\right)$, was measured at room temperature in the hope of determining whether $N\left(E_{F}\right)$ decreases with composition as the eutectic composition is approached. Finally, measurements of the superconducting transition temperature for these alloys were made as a function of $x$. These temperatures are compared, as a function of $z$, with other superconducting glasses to look for NFE behavior or charge-transfer effects.

\section{EXPERIMENTAL}

Buttons of $\mathrm{Zr}_{x} \mathrm{Pd}_{1-x}(0.59 \leqslant x \leqslant 0.70)$ were prepared by arc melting in a Centorr Series 5 furnace. The starting materials were $99.99 \%$-pure $\mathrm{Zr}$ rod and 99.99\%-pure Pd sponge. The furnace was operated using a continuous flow of prepurified argon gas. Approximately $5 \mathrm{~g}$ of sample were melted initially, with each button turned over and remelted four or more times to insure homogeneity. Based upon negligible weight changes observed after melting, the compositions were known to well within $1 \%$.

The amorphous samples were produced by melting about $0.1 \mathrm{~g}$ of alloy in a modified arc-melting furnace equipped with a copper plunger. ${ }^{16}$ The alloys were quenched into foils about $60-\mu \mathrm{m}$ thick by rapidly propelling the plunger onto the melted alloy. From the velocity of the plunger (almost $10 \mathrm{~m} / \mathrm{sec}$ ), a cooling rate of $10^{6}-10^{7} \mathrm{~K} / \mathrm{sec}$ was estimated. In order to insure the amorphous character of the samples, $\mathrm{x}$-ray diffraction measurements were performed. An example of a diffractogram, taken with $\mathrm{Cu} K \alpha$ radiation, is shown in Fig. 2. The first peak for the sample $\left(\mathrm{Zr}_{0.63} \mathrm{Pd}_{0.37}\right)$ occurs at $2 \theta=39 \mathrm{deg}$. At the relatively unsophisticated level of these measurements, all the compositions had similar structure factors. All measurements were made using as-quenched material.

The electrical-resistivity measurements were performed with standard four-probe techniques employ-

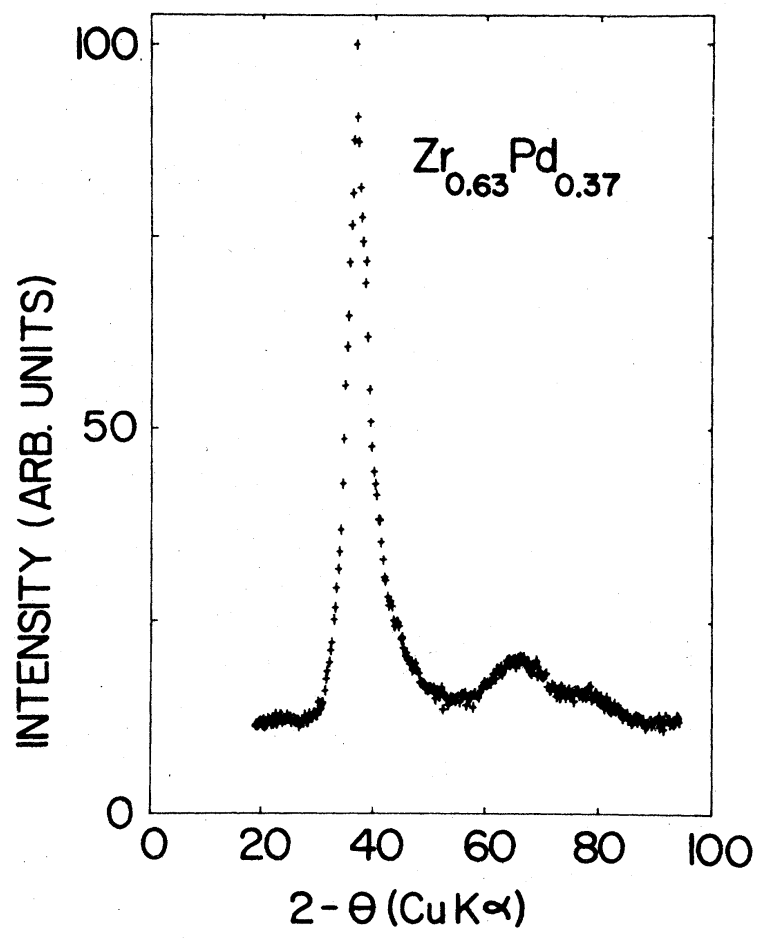

FIG. 2. X-ray diffractogram of amorphous $\mathrm{Zr}_{0.63} \mathrm{Pd}_{0.37}$.

ing a digital data-acquisition system. The current and potential leads were spot welded to the samples. Because the specimens were so thin, this was somewhat difficult. Good welds were obtained by first flattening that section of wire (about $2 \mathrm{~mm}$ ) being welded. Number $38 A W G$ copper wire was used. In the immediate vicinity of the welds, of course, the specimens were no longer amorphous; however, by cutting them and attaching their leads as shown in Fig. 3 , only the resistance of the amorphous material was measured.

The magnetic susceptibility was measured with a standard Faraday technique. ${ }^{17}$ Measurements were made only at room temperature to search qualitatively for concentration dependences in the Pauli susceptibilty and $N\left(E_{F}\right)$, as discussed in Sec. I.

\section{RESULTS AND DISCUSSION}

\section{A. Electrical resistivity}

Figure 4 shows the normalized resistivity $R(T)$ for three of the samples. Data below about $3 \mathrm{~K}$ are not shown because of the presence of superconducting transitions that develop there. These superconducting transitions are presumably the origin, through fluctuations, of the maxima seen in $R(T)$ at about $10 \mathrm{~K}$ for the three samples. The major points of Fig. 4 are that NTC values exist above $10 \mathrm{~K}$ for all samples, and that $R(T)$ changes only by a few percent 
for $3<T<300 \mathrm{~K}$. In this temperature range the resistivity of all samples is about $300 \mu \Omega \mathrm{cm}$. Several attempts were made, with different samples, to measure $R(T)$ for $\mathrm{Zr}_{0.59} \mathrm{Pd}_{0.41}$, but a smooth curve over the entire temperature range was not obtained. Discontinuities in the $R(T)$ data, which were not reproducible, occurred as the samples were heated and cooled. These jumps were of the order of $1 \%$ of $R(T)$. It seems likely that the specimens at this composition developed microscopic cracks when heated and cooled. Nevertheless, by piecing these data together, it was clear that $R(T)$ for this alloy was very similar to that exhibited by the other alloys.

With respect to theoretical models to account for the NTC, it must be noted that there is no region where $R(T)$ has a $\ln T$ temperature dependence. This Kondo-like dependence was suggested, by Cochrane et al., ${ }^{18}$ to arise from the presence of electron scattering from tunneling levels produced by atoms fluctuating between two configurations in the glass. In particular, this model gives

$$
\rho(T)=\rho_{0}-C \ln \left(T^{2}+\Delta^{2}\right),
$$

where $C$ is a constant and $\Delta$ is a temperature characteristic of the splitting between the two levels. Tsue ${ }^{19}$ has found several examples of metallic glasses for which Eq. (1) can fit the data, albeit with fairly high values of $\Delta$. Since Eq. (1) does not appear to be appropriate for $\mathrm{Zr}_{x} \mathrm{Pd}_{1-x}$, the extended $\mathrm{Zi}$ man theory as developed by Cote and Meisel, and $\mathrm{Nagel}^{12}$ was considered. Following Meisel and Cote, who have made detailed comparisons of this model

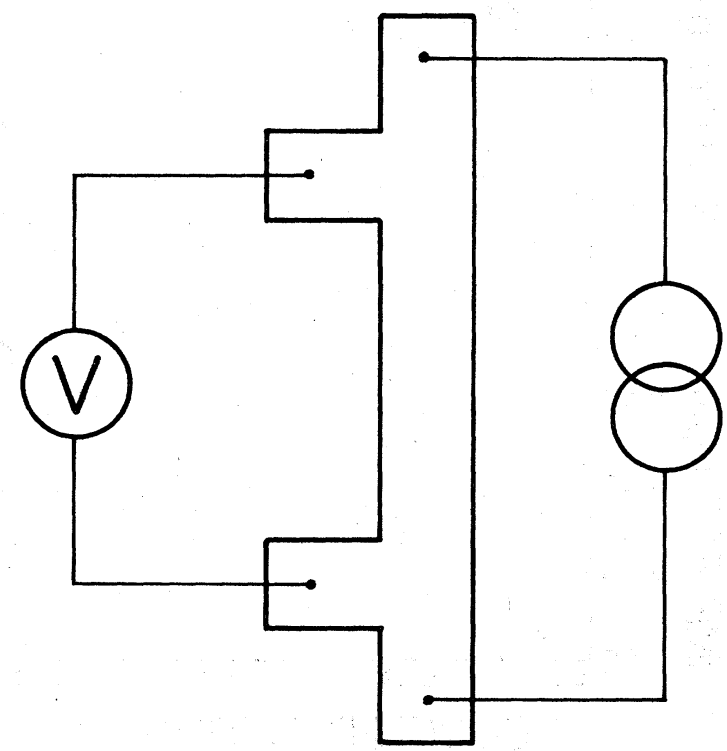

FIG. 3. Sketch showing shape of resistivity samples. By attaching the leads as shown, only the resistance of amorphous material is measured.

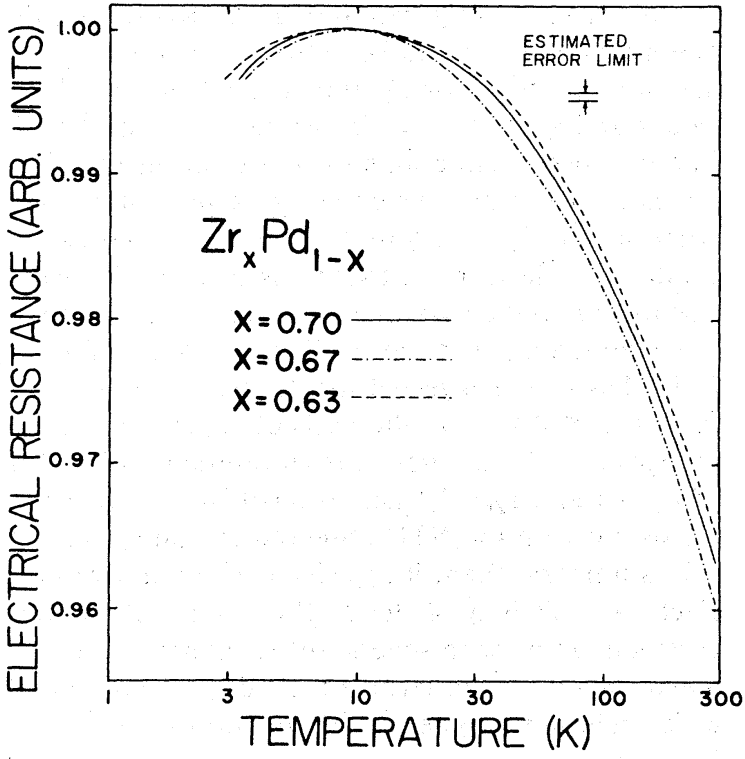

FIG. 4. Electrical resistance of $\mathrm{Zr}_{x} \mathrm{Pd}_{1-x}$ above about $3 \mathrm{~K}$.

with data on a number of systems, we shall in the following call this extended Ziman theory the diffraction model. In previous work we have shown that this model is consistent with $R(T)$ data on $\mathrm{Zr}_{0.4} \mathrm{Cu}_{0.6}{ }^{20}$ In addition, Clarke and $\mathrm{Nagel}^{21}$ have shown for another intertransition-metal glass, $\mathrm{Nb}_{0.6} \mathrm{Ni}_{0.4}$, that the NTC of $R(T)$ is consistent with the measured temperature dependence of the $\mathrm{X}$-ray structure factor $S(q)$, evaluated at the first peak $q_{p}$.

With respect to $\mathrm{Zr}_{x} \mathrm{Pd}_{1-x}$, the interesting question is: Can one rationalize $2 k_{F} \simeq q_{p}$, where $q_{p}$ is given by Fig. 2, in terms of reasonable $z$ values for $\mathrm{Zr}$ and $\mathrm{Pd}$ ? The detailed work of Meisel and Cote has shown that NTC are predicted by the diffraction model if $2 k_{F}$ is within about $10 \%$ of $q_{p}$. Values of the effective valence for $\mathrm{Zr}\left(z_{\mathrm{Zr}}\right)$ and $\mathrm{Pd}\left(z_{\mathrm{Pd}}\right)$ consistent with this criterion may be determined as follows. The $q_{p}$ value corresponding to $2 \theta=39 \mathrm{deg}$ is $q_{p}=2.72 \AA^{-1} . k_{F}$ is calculated from

$$
k_{F}=\left(3 \pi^{2} n\right)^{1 / 3},
$$

where $n$ is the conduction-electron density. $n$ is calculated by assuming that the density of glass (which has not been measured) is roughly the weighted average of the densities of crystalline $\mathrm{Zr}$ and $\mathrm{Pd}$; that is for $x=0.63, \bar{\rho}=8.53 \mathrm{~g} / \mathrm{cm}^{3}$. (The actual density is probably no more than a few percent less than this value.) The average number of conduction electrons for the glass is

$$
z_{\mathrm{eff}}=z_{\mathrm{Zr}} x+z_{\mathrm{Pd}}(1-x) .
$$

For elements like $\mathrm{Zr}$ and $\mathrm{Pd}$, it is by no means clear what the effective valences should be. Various authors ${ }^{22,23}$ have suggested that the late transition metals act as if they have $z$ values between 0.5 and 1.0 . 
The electronic configuration of $\mathrm{Zr}$ is $[\mathrm{Kr}] 4 d^{2} 5 s^{2}$; thus one might expect $z_{\mathrm{Zr}}$ to be between 2 and 4 . Assuming various values for $z_{\mathrm{Zr}}$ and $z_{\mathrm{Pd}}$ leads to the $2 k_{F}$ values and $\eta \equiv\left(2 k_{F}-q_{p}\right) / q_{p}$ values listed in Table I. The first two assumptions for the valences of $\mathrm{Zr}$ and Pd leads to $\eta$ values which do not satisfy Meisel-Cote criterion for NTC $(\eta \leqslant 0.1)$. The table suggests that conduction-electron contributions of 1 or somewhat less for $\mathrm{Pd}$, and approximately 2 for $\mathrm{Zr}$, are required to be consistent with the $R(T)$ data. A $z_{\mathrm{Zr}}$ of about 2 also has been suggested for $\mathrm{Be}_{x} \mathrm{Zr}_{1-x}$ glasses $(0.30 \leqslant x \leqslant 0.45)$, on the basis of superconducting properties. ${ }^{24}$ In addition, the assumption in $\mathrm{Zr}_{0.4} \mathrm{Cu}_{0.6}$ of $z_{\mathrm{Cu}}=1$ and $z_{\mathrm{Zr}}=2$ leads to $\eta=0.04$, which again is consistent with the NTC observed in that system.

In summary, then, it appears as if the normal-state electrical resistivity of the $\mathrm{Zr}_{x} \mathrm{Pd}_{1-x}$ glasses can be understood, along with several other intertransitionmetal glasses, ${ }^{20,25}$ in terms of the diffraction model which relies on a spherical Fermi surface with $2 k_{F} \approx q_{p}$.

\section{B. Magnetic susceptibility}

In this section we describe an attempt to determine whether $N\left(E_{F}\right)$ decreases as the eutectic composition is approached. Again, if the electronic factors are important for glass formation, and if they are at the root of the abnormally deep eutectics seen in many of the phase diagrams, then such a correlation might exist, as suggested in the work of Nagel and Tauc. ${ }^{4}$ The other assumption that must be made is that the Pauli susceptibility is given by

$$
\chi_{p}=2 \mu_{\mathrm{B}}^{2} N\left(E_{F}\right) S,
$$

where $S$, the Stoner enhancement factor, is given by

$$
S=\left[1-J_{\text {eff }} N\left(E_{F}\right)\right]^{-1},
$$

$J_{\text {eff }}$ being an effective exchange interaction. Assuming that $S$ is approximately independent of $x$, then any structure or trends observed in $\chi_{p}(x)$. would be related to corresponding structure in $N\left(E_{F}(x)\right)$.

The relation between the measured susceptibility and $X_{p}$ is complicated by at least two effects. One is that the diamagnetic contribution from the cores should be removed. This contribution should change smoothly with $x$ in a straightforward way. However, a much more serious problem in using $X_{p}(x)$ to gauge $N\left(E_{F}(x)\right)$ is that the effect of unwanted paramagnetic impurities must be removed. This can be attempted by measuring the susceptibility as a function of temperature and removing a Curie-like term, or by using the Honda-Owen technique. ${ }^{26}$ There are uncertainties in both methods, especially in alloys containing $\mathrm{Pd}$, but we have used the HondaOwen method because it obviates lengthy measurements as a function of temperature.
TABLE I. $2 k_{F}$ vlues and $\eta \equiv\left(2 k_{F}-q_{p}\right) / q_{p}$ values.

\begin{tabular}{llcc}
\hline & \multicolumn{3}{c}{$\mathrm{Zr}_{0.63}{ }^{2} d_{0.37}$ glass } \\
$2 k_{F}\left(\AA^{-1}\right)$ & $\eta$ \\
$z_{\mathrm{Zr}}$ & $z_{\mathrm{Pd}}$ & 3.31 & 0.22 \\
\hline 4 & 1 & 3.24 & 0.19 \\
4 & 0.5 & 2.74 & 0.01 \\
2 & 1 & \\
\hline
\end{tabular}

Using the Honda-Owen technique, one assumes that the sample contains only saturated magnetic impurities of total moment $\mu_{s}$. The measured Faraday susceptibility $\chi_{m}$ is then related to the pure-sample susceptibility $\chi$ as

$$
\chi_{m}=\chi+\mu_{s} / H
$$

Thus, by plotting $\chi_{m}$ vs $H^{-1}$, both $\chi$ and $\mu_{s}$ can be estimated. Figure 5 shows the susceptibilities determined from room-temperature Honda-Owen plots. The data were taken in fields up to $10.4 \mathrm{kOe}$. The reason for the downward arrows in the figure (belonging to the susceptibility data) is that a field of $10.4 \mathrm{kOe}$ may not have been large enough to saturate all the impurity moments, whence the plotted values of $\chi$ may be only upper bounds to the host susceptibility. (This is an intrinsic limitation of the HondaOwen technique.) Estimates of the ferromagneticimpurity concentrations, also shown in Fig. 5, were determined from the Honda-Owen plots assuming a

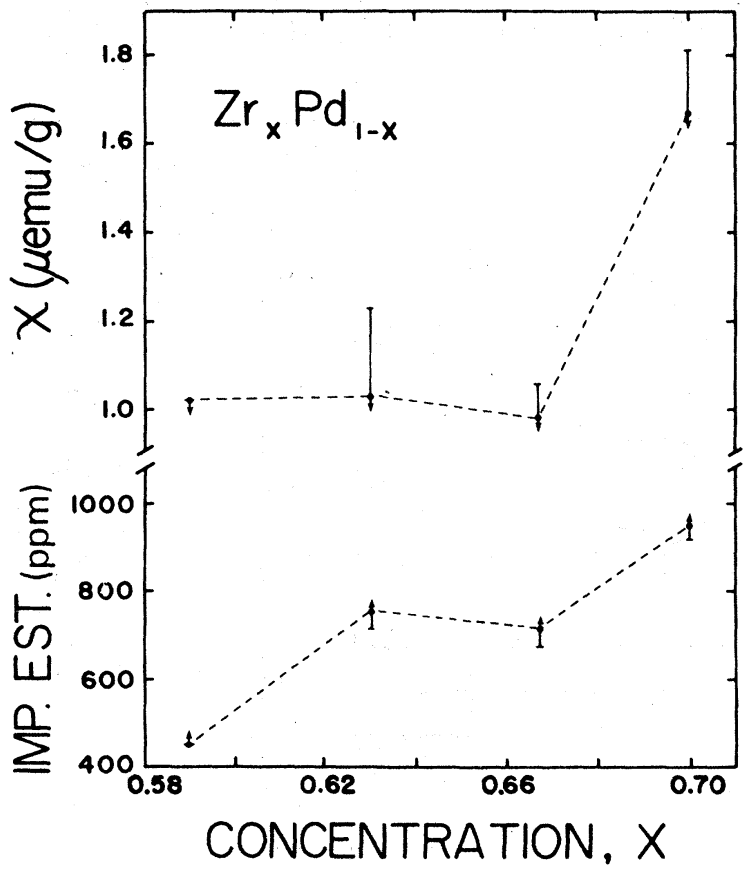

FIG. 5. Susceptibility $\chi$ and estimates of the ferromagneticimpurity content (Imp. Est.) vs concentration for $\mathrm{Zr}_{x} \mathrm{Pd}_{1-x}$. Data determined from Honda-Owen plots. 
TABLE II. Superconducting transition temperatures and widths for $\mathrm{Zr}_{x} \mathrm{Pd}_{1-x}$.

\begin{tabular}{ccc}
\hline$x$ & $T_{c}(\mathrm{~K})^{\mathrm{a}}$ & $\Delta T_{c}(\mathrm{~K})^{\mathrm{b}}$ \\
\hline 0.59 & 1.76 & $\leqslant 0.2$ \\
0.63 & 2.20 & $\leqslant 0.06$ \\
0.67 & 2.37 & $\leqslant 0.03$ \\
0.70 & 2.66 & $\leqslant 0.03$ \\
\hline
\end{tabular}

a Uncertainties in $T_{c}$ are approximately $\pm 0.08 \mathrm{~K}$.

${ }^{\mathrm{b}} \Delta T_{c}$ was estimated on the basis of 10 to $90 \%$ of the normal-state resistance.

saturation magnetization contribution of $1 \mu_{\mathrm{B}}$ per impurity atom. The upward arrows indicate that the estimates are too low if the impurity moments did not saturate. The impurity content indicated is higher than that suggested by the nominal materials analyses and, because $\chi(x)$ crudely scales with the estimated impurity content, it is possible that the data may not be a good measure of $N\left(E_{F}\right)$.

It is clear from Fig. 5 that $\chi(x)$ does not decrease as the eutectic composition is approached, i.e., as $x$ increases. ${ }^{27}$ In fact, if any trend exists, it is that $\chi(x)$ increases with $x$. If this result is not an artifact of magnetic impurities, then it appears that $N\left(E_{F}\right)$ increases as the eutectic composition is approached. It follows that if there does exist a stabilization of the liquid owing to a lowering of the electronic density of states, then this stabilization factor is not maximized at a concentration near that of the eutectic and, perhaps, has a small effect on the liquid-glassforming tendency. Systematic measurements involving, for example, soft-x-ray and photoemission ${ }^{28}$ studies, would be useful in determining whether a correlation exists between $N\left(E_{F}\right)$ and the ease of glass formation. It should be mentioned that Mizutani et al. ${ }^{29}$ recently have reported low-temperature specific-heat measurements on Pd-Si alloys which indicate that there exists a maximum in $N\left(E_{F}\right)$ near the eutectic composition.

\section{Superconductivity}

Table II lists the superconducting transition temperatures $T_{c}$ and widths $\Delta T_{c}$ for the four concentrations. For $x=0.70$, the $T_{c}$ measured is slightly higher than that measured by Johnson and Poon (2.4 K) ${ }^{30}$ or by Graebner et al. $(2.53 \mathrm{~K}){ }^{31}$ Figure 6 shows $T_{c}$ as a function of $x$ or the $z$ ratio. This ratio was calculated by assuming $z_{\mathrm{Zr}}=4$ and $z_{\mathrm{Pd}}=10$, so that the resulting $T_{c}$ curve could readily be compared with the Collver-Hammond curve. ${ }^{15}$ (As discussed in Sec. III.A, these $z$ values may not have any fundamental significance.) The $T_{c}$ values scale linearly with $x$ (and $z$ ), but fall below the $T_{c}(z)$ curve of
Collver and Hammond. Johnson and Poon, ${ }^{30}$ Collver and Hammond, ${ }^{32}$ and Hasegawa and Tanner ${ }^{24}$ have measured $T_{c}$ for a variety of amorphous superconductors and the pattern of behavior that is becoming established seems to be that, for amorphous materials composed of transition metals widely separated in z. $T_{c}(\mathbf{z})$ lies near or below the Collver-Hammond curve for close-neighbor elements. Collver and Hammond $^{32}$ have suggested that this decrease in $T_{c}$ may be due to a transfer of charge from the more electropositive to the more electronegative elements, e.g., from $\mathrm{Zr}$ to $\mathrm{Pd}$. It should be noted that in the case of Be-Zr glasses, Hasegawa and Tanner ${ }^{24}$ concluded that the increase of $T_{c}$ with $\mathrm{Zr}$ content was due, not to a change in the electronic structure, but rather to an increase in structural disorder. Additional measurements on both relaxed and as-quenched glasses may be useful in assessing the effects of charge transfer, real-space structure, and electronic structure on the superconductivity of metallic glasses.

\section{SUMMARY AND CONCLUSIONS}

The major results of this study can be summarized as follows:

(i) $\mathrm{Zr}_{x} \mathrm{Pd}_{1-x}$ glasses exhibit negative temperature coefficients of the resistivity between about 10 and $300 \mathrm{~K}$. If conduction-electron contributions of about 2 and about 1 are assumed for $\mathrm{Zr}$ and $\mathrm{Pd}$, respective$l y$, then the data can be rationalized in terms of the diffraction model for metallic glasses. This relies on a spherical Fermi surface with $2 k_{F} \simeq q_{p}$.

(ii) $N\left(E_{F}\right)$ does not seem to decrease with composition as the eutectic composition is approached. This result does not lend any support to the idea that the energy of the conduction-electron system is connected in a simple way with the thermodynamic stability of the liquid state, and thus to the ease of glass formation of a liquid.

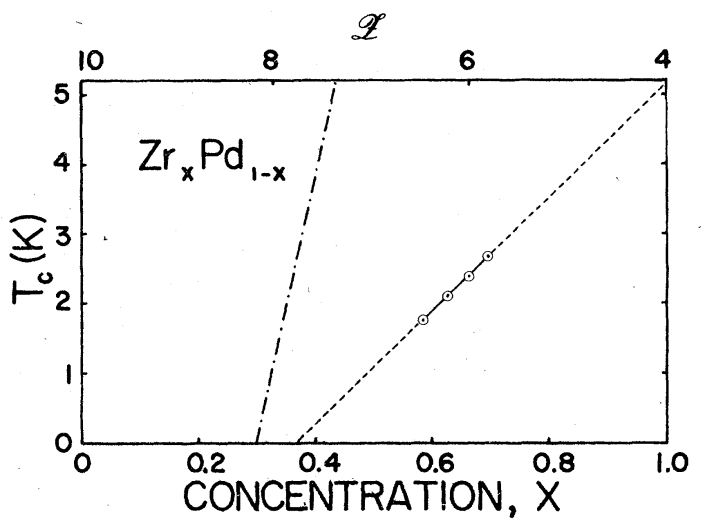

FIG. 6. Superconducting transition temperatures for $\mathrm{Zr}_{x} \mathrm{Pd}_{1-x}$. The dashed-dotted line is a portion of that curve initially determined by Collver and Hammond (Ref. 15). 
(iii) The concentration dependence of the superconducting transition temperature was found to increase linearly with $x$. However, the $T_{c}$ values do not correlate in a simple way with previous measurements on amorphous alloys composed of near neighbors in the Periodic Table. The observed rounding of the superconducting transition over a relatively large temperature region probably is due to superconducting fluctuations above $T_{c}$ and reflects a very small coherence length.

In conclusion, the present results suggest that the most critical need to further the understanding of intertransition-metal alloys is information on charge transfer, Fermi-surface diameters, and electronic structure in general. Measurements such as positron annihilation, soft $x$-ray spectroscopy, and photoemission should be of great interest for this class of materials.

\section{ACKNOWLEDGMENTS}

We are grateful to John Weymouth and William Burmester for assistance with the measurements and helpful discussions. We are indebted to Frans Spaepen and David Turnbull for their critical reading of the manuscript. Support of this work by NSF Grant No. DMR76-17417 is gratefully acknowledged.
"Present Address: Division of Applied Sciences, Harvard University, Cambridge, Ma. 02135

${ }^{1}$ For a recent review and references, see: P. Chaudhari and D. Turnbull, Science 199, 11 (1978).

${ }^{2}$ D. E. Polk, Acta Matall. 20, 485 (1972)

${ }^{3}$ H. S. Chen and B. K. Park, Acta Metall. 21, 395 (1973).

${ }^{4}$ S. R. Nagel and J. Tauc, Phys. Rev. Lett. $\underline{35}, 380$ (1975).

${ }^{5}$ W. Bauhofer and A. Simon, Phys. Rev. Lett. 40, 1730 (1978)

${ }^{6}$ T. M. Hayes, J. W. Allen, J. Tauc, B. C. Giessen, and J. J Hauser, Phys. Rev. Lett. 40, 1282 (1978).

${ }^{7}$ D. Turnbull, J. Phys. (Paris), 35, 1 (1974), Chap. 4.

${ }^{8}$ Frans Spaepen, in Proceedings of the 3rd International Conference on Rapidly Quenched Metals (to be published).

${ }^{9}$ S. R. Nagel and J. Tauc, Solid State Commun. 21, 129 (1977).

${ }^{10} \mathrm{M}$. Norman and I. R. Harris, J. Less Common Metals $\underline{18}$, 333 (1969); see also A. R. Miedema, F. R. de Boer, and P. F. de Chatel, J. Phys. F 3 , 1559 (1973).

${ }^{11} \mathrm{M}$. Marcus and D. Turnbull, J. Mat. Sci. Eng. 23, 211 (1976).

${ }^{12}$ See: P. J. Cote and L. V. Meisel, Phys. Rev. Lett. 40, 1586 (1978); L. V. Meisel and P. J. Cote, Phys. Rev. B (in press); S. R. Nagel, Phys. Rev. B 16, 1694 (1977), and references therein.

${ }^{13}$ W. Buckel and R. Hilsch, Z. Phys. 138, 109 (1954).

${ }^{14}$ For a recent review, see William L. Johnson, in Proceedings of the 3rd International Conference on Rapidly Quenched Metals, (to be published); and G. Bergmann, Phys. Rep. 27, 159 (1976).

${ }^{15}$ M. M. Collver and R. H. Hammond, Phys. Rev. Lett. 30, 92 (1973).
${ }^{16}$ The apparatus is similar to that of M. Ohring and A. Haldipur, Rev. Sci. Instrum. 42, 530 (1971).

${ }^{17}$ See, e.g., C. M. Hurd, Cryogenics 6, 264 (1966).

${ }^{18} \mathrm{R}$. W. Cochrane, R. Harris, J. O. Strom-Olson, and M. J. Zuckermann, Phys. Rev. Lett. 35, 676 (1975).

${ }^{19} \mathrm{C}$. C. Tsuei, Bull. Am. Phys. Soc. 22, 322 (1977); 23, 406 (1978); 23, 359 (1978)

${ }^{20}$ F. R. Szofran, G. R. Gruzalski, J. W. Weymouth, D. J. Sellmyer, and B. C. Giessen, Phys. Rev. B 14, 2160 (1976).

${ }^{21}$ R. Clarke and S. R. Nagel (unpublished).

22p. J. Cote, Solid State Commun. 18, 1311 (1976)

${ }^{23} \mathrm{G}$. Busch and H.-J. Güntherodt, Solid State Phys. 29, 235 (1974).

${ }^{24}$ R. Hasegawa and L. E. Tanner, Phys. Rev. B 16, 3925 (1977).

${ }^{25}$ S. R. Nagel, J. Vassiliov, P. M. Horn, and B. C. Giessen, Phys. Rev. B 17, 462 (1978).

${ }^{26}$ See, for example, L. F. Bates, Modern Magnetism, 4th ed (Cambridge University, London, 1961).

${ }^{27}$ For a phase diagram, see R. P. Elliott, Constitution of Binary Alloys, First Supplement (McGraw-Hill, New York, 1965)

${ }^{28}$ For the Nb-Ni system, see: S. R. Nagel, J. Tauc, and B. C. Giessen, Solid State Commun. 22, 471 (1977).

${ }^{29}$ U. Mizutani, K. T. Hartwig, and T. B. Massalski, Phys. Rev. Lett. 41,661 (1978)

${ }^{30}$ W. L. Johnson and S. J. Poon, J. Appl. Phys. 46, 1787 (1975).

${ }^{31}$ J. E. Graebner, B. Golding, R. J. Schutz, F. S. L. Hsu, and H. S. Chen, Phys. Rev. Lett. 39, 1480 (1977).

${ }^{32}$ M. M. Collver and R. H. Hammond, Solid State Commun. 22, 55 (1977) 